\title{
Liderazgo transformacional en las relaciones interpersonales y trabajo colaborativo de los directivos
}

\author{
Transformational leadership in interpersonal relationships and collaborative work of managers
}

Liderança transformacional nas relações interpessoais e no trabalho colaborativo dos gerentes

Dennis Fernando Jaramillo Ostos

dennisfernan@hotmail.com

https://orcid.org/0000-0003-0432-7855

Universidad César Vallejo, Lima-Perú

Universidad Científica del Sur, Lima-Perú

\author{
Luz Marina Acero Coaquira \\ laceroc@unap.edu.pe \\ https://orcid.org/0000-0002-2355-524X
}

Universidad Nacional del Altiplano, Perú
William Jesús Rojas Gutiérrez
williamtor@hotmail.com
https://orcid.org/0000-0001-5296-2971

Universidad Privada San Juan Bautista, Lima-Perú

Alejandro Sabino Menacho Rivera

alejandro.131071@gmail.com

https://orcid.org/0000-0003-2365-8932

Universidad César Vallejo, Lima-Perú

Artículo recibido en junio 2021, revisado en julio 2021, arbitrado en agosto 2021 y publicado en septiembre 2021

\section{RESUMEN}

El estudio tuvo como objetivo determinar la incidencia que tiene el liderazgo transformacional en las relaciones interpersonales y trabajo colaborativo de los directivos de la UGEL 11. El estudio fue de tipo básico, el método hipotético deductivo y el diseño no experimental, la población de estudio comprendió 380 individuos entre docentes, directivos, personal de servicio y administrativo de la UGEL 11. La muestra fue de 75 individuos, siendo el muestreo no probabilístico. La técnica empleada fue la encuesta en la cual se colectó la pesquisa usando un muestreo no probabilístico; como instrumento se empleó el cuestionario que fue validado por juicio de expertos y con una prueba binomial para las tres variables, estas pruebas validaron los instrumentos. Según los resultados obtenidos se concluye que el liderazgo transformacional incide significativamente en las relaciones interpersonales y trabajo colaborativo en las instituciones educativas de la UGEL 11 Cajatambo.

Palabras clave: Motivación docente; Acompañamiento docente; Trabajo en equipo

\section{ABSTRACT}

The objective of the study was to determine the impact that transformational leadership has on interpersonal relationships and collaborative work of the UGEL 11 managers. The study was of a basic type, the hypothetical deductive method and the non-experimental design, the study population included 380 individuals among teachers, directors, service and administrative staff of UGEL 11. The sample was 75 individuals, the sampling being non-probabilistic. The technique used was the survey in which the research was collected using a nonprobabilistic sampling; As an instrument, the questionnaire was used, which was validated by expert judgment and with a binomial test for the three variables, these tests validated the instruments. According to the results obtained, it is concluded that transformational leadership has a significant impact on interpersonal relationships and collaborative work in the educational institutions of UGEL 11 Cajatambo.

Key words: Teacher motivation; Teacher support; Teamwork

\section{RESUMO}

O objetivo do estudo foi verificar o impacto que a liderança transformacional tem nas relações interpessoais e no trabalho colaborativo dos gestores da UGEL 11. $\mathrm{O}$ estudo foi do tipo básico, o método hipotético dedutivo e o delineamento não experimental, a população do estudo compreendeu 380 indivíduos entre professores, diretores, funcionários de serviço e administrativos da UGEL 11. A amostra foi de 75 indivíduos, sendo a amostra não probabilística. A técnica utilizada foi a survey em que a pesquisa foi coletada por meio de amostragem não probabilística; Como instrumento, foi utilizado o questionário, o qual foi validado por julgamento de especialistas e com um teste binomial para as três variáveis, esses testes validaram os instrumentos. De acordo com os resultados obtidos, concluise que a liderança transformacional tem um impacto significativo nas relações interpessoais e no trabalho colaborativo nas instituições de ensino da UGEL 11 Cajatambo.

Palavras-chave: Motivação do professor; Apoio ao professor; Trabalho em equipe 


\section{INTRODUCCIÓN}

El líder motiva y dirige a un grupo de individuos con la finalidad de lograr cumplir objetivos en común. El liderazgo es aplicado en diferentes ámbitos. Es así que, en el ámbito empresarial, ser líder significa tener la dirección de los trabajadores o servidores llevando a cabo una estrategia que permita la satisfacción de las necesidades organizacionales. Para ser un buen líder, el individuo debe desarrollar diferentes habilidades que permitan la buena interacción y mentoría al equipo de trabajo, teniendo como una de sus metas que cada integrante logre más de lo que se espera, creativa y activamente (Alqatawenah, 2018).

En el ámbito educativo, el liderazgo se asocia a las competencias del director para impactar positivamente en el personal docente, con la finalidad de mejorar el uso de las técnicas y métodos pedagógicos. Este proceso debe estar sometido a una retroalimentación permanente que se acople a los diferentes contextos que se presentan; es por esto que cuando los directivos plantean desarrollar proyectos educativos, requieren de la participación e involucramiento del equipo en general, a fin de que estos proyectos sean productivos y sostenibles (Rosas, 2020).

En este sentido, para lograr el desarrollo de habilidades organizacionales y de gestión uno de los factores importantes es el liderazgo pedagógico, ya que este compromete, motiva e incentiva al equipo educativo a desarrollar un trabajo colaborativo, aprender en grupo y a desarrollarse de manera democrática, respetándose entre sí y trabajando hacia un objetivo en común (Contreras, 2016). Teniendo en cuenta este enfoque, la manera en que las instituciones educativas podrían obtener mejores resultados académicos y de esta manera ofrecer un desarrollo integral docente, dependería de los directivos a cargo y de cómo estos ejecutan su liderazgo, esperando que se motive al equipo a la mejora continua y a su desarrollo como profesionales (Rodríguez, et al., 2020).

El Ministerio de Educación en Perú demanda el involucramiento permanente de los directivos de las instituciones educativas, con la finalidad de que se pueda asegurar la alineación de los docentes a los objetivos planteados por el ministerio. De esta manera conseguir que los estudiantes puedan desarrollarse integralmente teniendo en cuenta el Diseño Curricular Nacional (Ministerio de Educación, 2018).

Para poder desarrollar los objetivos planteados, es necesario que los integrantes del equipo educativo cuenten con buenas relaciones interpersonales y sean capaces de trabajar de forma colaborativa, sin embargo, a nivel local, en muchas de las instituciones educativas de la provincia de Cajatambo, se presentan problemas de coordinación entre los colaboradores, además, la cooperación de parte de los directivos es nula o muy baja; lo que no permite que se desarrolle un trabajo colaborativo, afectando incluso el clima laboral.

Bajo estos aspectos, se formuló la siguiente pregunta de investigación ¿cómo incide un liderazgo transformacional en las relaciones interpersonales y el trabajo colaborativo en los docentes de las instituciones educativas de la UGEL $\mathrm{N}^{\circ} 11$ de Cajatambo? Por otro lado, los problemas específicos comprendieron la determinación de la incidencia de las dimensiones consideración individual, motivación y estimulación intelectual en las relaciones interpersonales y trabajo colaborativo de los directivos.

El liderazgo es descrito por el autor Madrigal (2011) como la influencia que tiene el líder sobre su equipo a través de conductas y/o habilidades que aumentan la motivación y ayudan al individuo o grupo a cumplir los objetivos planteados en 
conjunto. En relación al liderazgo transformacional, el líder muestra diversos patrones a seguir en relación a determinadas circunstancias. El líder da la motivación a su equipo para lograr los objetivos en común para el beneficio de todos (Avolio y Bass, 2004).

Según los autores Avolio y Bass (2004), se deben de incluir los siguientes factores para poder implementar el liderazgo transformacional en una organización: El factor motivación, describe la capacidad que tiene el líder para brindar motivación a su equipo con la finalidad de obtener un buen clima organizacional y lograr los objetivos propuestos. El factor influencia idealizada, hace referencia a la influencia que tiene el líder de una manera idealizada, brindando un propósito a su equipo. La consideración Individual, referente al respeto a la individualidad de cada integrante del equipo, teniendo en consideración la empatía y cuidado al proponer retos al grupo. El factor estimulación intelectual, referente al empoderamiento estimulado por el líder para que los integrantes de su equipo desarrollen sus habilidades intelectuales.

Respecto a la variable relaciones interpersonales, Dalton, Hoyle, y Watts (2007) lo definen como una de las habilidades innatas del ser humano, la cual se desarrolla y perfecciona a lo largo del tiempo, esta habilidad es determinante para lograr la autoconfianza y la confianza entre los miembros de un equipo, a fin de que se coexistencia sea satisfactoria y eficiente se necesita dos aspectos: El compromiso organizacional, que haced referencia a cuando los trabajadores de la organización muestran un desempeño eficaz y dedicación a su trabajo. El segundo aspecto es la habilidad comunicativa relacionada a la interacción dentro del equipo, cultivando el respeto y comprensión del grupo.
Respecto a la variable trabajo colaborativo los autores Driscoll y Vergara (1997) los definen como un trabajo en equipo que genera su crecimiento y desarrollo propio, respetando a cada uno de sus integrantes, extrayendo aprendizajes y reflexiones desde la experiencia compartida. Para esto es necesario tener en cuenta componentes como la interdependencia, el desarrollo grupal y la responsabilidad.

El presente estudio, desde una perspectiva práctica, se justifica porque permite el acceso a un diagnóstico de la situación actualizada de la institución educativa, pues persigue la evaluación de alternativas de mejora y la más provechosa toma de decisiones. Epistemológicamente cobra relevancia porque describe el contexto de las instituciones educativas, resaltando sus características y la realidad problemática de las instituciones.

A consecuencia de lo argumentado anteriormente, el estudio tuvo como objetivo determinar la incidencia que tiene el liderazgo transformacional en las relaciones interpersonales y trabajo colaborativo de los directivos de la UGEL $\mathrm{N}^{\circ}$ 11. Por otro lado, los objetivos específicos se centraron en establecer la incidencia de la influencia idealizada en las relaciones interpersonales y trabajo colaborativo de los directivos de la UGEL 11.

\section{MÉTODO}

El estudio fue de tipo básico, ya que fueron utilizadas las teorías de las variables con la finalidad de crear conocimientos nuevos. El método fue hipotético deductivo, ya que no hubo manipulación deliberada de alguna variable. (Hernández y Mendoza, 2018). La finalidad de la investigación fue probar si es que el liderazgo transformacional incidía en las relaciones interpersonales y en el trabajo 
colaborativo. El estudio fue de corte transversal, ya que los datos fueron obtenidos en un determinado momento. La población de estudio comprendió 380 individuos entre docentes, directivos, personal de servicio y administrativo; quienes laboran dentro de la UGEL $\mathrm{N}^{\circ} 11$ en el distrito de Cajatambo. La muestra estuvo conformada por 75 individuos, siendo el muestreo no probabilístico.

La técnica empleada fue la encuesta en la cual se colectó la pesquisa usando un muestreo no probabilístico; como instrumento se utilizó el cuestionario el cual fue validado por juicio de expertos, además la validación de constructo se dio con una prueba binomial para las tres variables, estas pruebas validaron los instrumentos. Para el cuestionario de relaciones interpersonales estuvo constituido por 28 ítems en los que se consideró las dimensiones habilidades comunicativas $y$ compromiso organizacional. El cuestionario de trabajo colaborativo constó de 30 ítems y tomó en cuenta las dimensiones proceso de grupo, habilidades de colaboración, interacción promotora, interdependencia positiva y responsabilidad individual.

El cuestionario de Liderazgo transformacional constó de 20 ítems en las que de considero las dimensiones influencia idealizada, motivación inspiracional, estimulación intelectual y consideración individual donde las preguntas fueron dirigidas a la percepción que se tienen de los directivos según el rol que cumplen en sus instituciones educativas.
Para determinarla confiabilidad, se realizó una prueba piloto con cada instrumento a través del Alfa de Cronbach, obteniendo 0,938 ; 0,945 y 0,943 para las variables liderazgo transformacional, relaciones interpersonales y trabajo colaborativo, respectivamente; estos resultados representan un alto nivel de confiabilidad.

El instrumento fue aplicado de manera virtual utilizando Google Forms, los datos obtenidos fueron procesados, se contrastó la hipótesis usando el estadístico de regresión ordinal simple, se tomaron en cuenta las dimensiones consideración individual, motivación y estimulación intelectual para determinar su influencia en las relaciones interpersonales y trabajo colaborativo de los directivos.

\section{RESULTADOS Y DISCUSIÓN}

\section{Prueba de hipótesis general}

Según los resultados de la regresión del Pseudo R cuadrado, el liderazgo transformacional, influye en las variables relaciones interpersonales y trabajo colaborativo de los directivos de la UGEL 11-Cajatambo, ya que el coeficiente de Nagelkerke indica que las buenas o malas relaciones interpersonales y trabajos colaborativos, se deben en un $72,5 \%$ al liderazgo implementado en las instituciones educativas (ver Tabla 1).

Tabla 1. Resultado de regresión por Pseudo R-cuadrado.

Pseudo R cuadrado

$\begin{array}{llllll}\text { Cox y Snell } & \text { N574 } & \text { Nagelkerke } & \text { McFadden } & , 534\end{array}$

Función de enlace: Logit. 


\section{Prueba de hipótesis específicas}

Prueba de hipótesis específica 1

A partir de los resultados obtenidos por el $\mathrm{R}$ del Pseudo cuadrado, el coeficiente de Nagelkerke se afirma que el 63,7\% del actuar de la consideración individual implementadas en las instituciones educativas, influyen en el trabajo colaborativo y las relaciones interpersonales de los directivos de la UGEL 11 - Cajatambo. En decir si no hay un buen actuar de la consideración individual, estas relaciones serán inestables (ver Tabla 2).

Tabla 2. Resultado de regresión por Pseudo R-cuadrado, dimensión Consideración Individual.

\begin{tabular}{llllll}
\hline Cox y Snell &, 504 & Nagelkerke &, 637 & McFadden &, 417 \\
\hline
\end{tabular}

Función de enlace: Logit.

Además, respecto a las derivaciones se observa el resultado de la regresión respecto al trabajo colaborativo nivel 1 (Bajo) y las relaciones interpersonales también de nivel 1 (Bajo). Por otro lado, la conducta del trabajo colaborativo es inseguridad con un coeficiente de $-22,730$ y las relaciones interpersonales es complicación con 0.435 . En consecuencia, si hay una mala práctica de la consideración individual, las relaciones interpersonales y el trabajo colaborativo serán bajos los directivos de la UGEL 11 - Cajatambo. Probándose estos con la prueba de Wald donde se obtuvo 810.145 y 529.878 respectivamente, (ver Tabla 3). Además, el p_value es menor a 0.05 por lo que se acepta la hipótesis alterna $\mathrm{H} 1=\mathrm{La}$ consideración individual tiene incidencia en el trabajo colaborativo y las relaciones interpersonales de los directivos de la UGEL 11, Cajatambo.

Tabla 3. Coeficientes de la consideración individual en las relaciones interpersonales y el trabajo colaborativo. Estimaciones de parámetro.

\begin{tabular}{|c|c|c|c|c|c|c|c|c|}
\hline & & \multirow{2}{*}{ Estimación } & \multirow{2}{*}{$\begin{array}{l}\text { Error } \\
\text { estándar }\end{array}$} & \multirow{2}{*}{ Wald } & \multirow{2}{*}{ Gl } & \multirow{2}{*}{ Sig. } & \multicolumn{2}{|c|}{$\begin{array}{c}\text { Intervalo de } \\
\text { confianza al 95\% }\end{array}$} \\
\hline & & & & & & & $\begin{array}{l}\text { Límite } \\
\text { inferior }\end{array}$ & $\begin{array}{l}\text { Límite } \\
\text { superior }\end{array}$ \\
\hline \multirow[t]{2}{*}{ Umbral } & {$[$ Consid_Indiv $=1]$} & $-22,956$ & 529,878 & 1,172 & 1 & ,000 & $-2,687$ & ,775 \\
\hline & {$[$ Consid_Indiv $=2]$} & $-20,387$ & 354,893 & 2,412 & 1 &, 000 &,- 363 & 3,138 \\
\hline \multirow[t]{6}{*}{ Ubicación } & [Relac_intepr=1] & $-19,378$ & 810,145 & 1,458 & 1 & ,000 & $-3,615$ & ,859 \\
\hline & [Relac_intepr=2] & $-22,730$ & 1,101 & ,427 & 1 & . & $-2,879$ & 1,439 \\
\hline & [Relac_intepr=3] & $0 \mathrm{a}$ & . & . & 0 & . & . & . \\
\hline & {$[$ trab_colab $=1]$} & ,435 & ,922 & 2,414 & 1 & ,708 &,- 375 & 3,238 \\
\hline & {$[$ trab_colab=2] } & ,046 & ,917 & 1,301 & 1 & ,713 &,- 751 & 2,843 \\
\hline & {$[$ trab_colab=3] } & $0 \mathrm{a}$ & . & . & 0 & . & . & . \\
\hline
\end{tabular}

Función de enlace: Logit.

a. Este parámetro está establecido en cero porque es redundante. 


\section{Prueba de hipótesis específica 2}

Desde los resultados obtenidos por el $\mathrm{R}$ del Pseudo cuadrado, se aprecia que la estimulación intelectual influye en en las relaciones interpersonales y el trabajo colaborativo en los directivos de la UGEL 11 - Cajatambo, además el coeficiente de Nagelkerke refiere que $67,5 \%$ del actuar de la consideración individual influye en el trabajo colaborativo y las relaciones interpersonales (ver Tabla 4).

Tabla 4. Resultado de regresión por Pseudo R-cuadrado, dimensión estimulación intelectual.

\begin{tabular}{llllll}
\hline Cox y Snell &, 560 & Nagelkerke &, 675 & McFadden &, 487 \\
\hline
\end{tabular}

Función de enlace: Logit.

Respecto a los coeficientes de la regresión, referente a la conducta de las relaciones interpersonales es complicación presentando un coeficiente 0.220 y el trabajo colaborativo es inseguridad con $-22,343$ concluyendo que si se tienen malas prácticas de estimulación intelectual se tendrá bajas relaciones personales y trabajo colaborativo en los directivos de la UGEL 11 -
Cajatambo. Probándose estos con la prueba de Wald donde se obtuvo 893.508 y 578.186 (ver Tabla 5).

Además, el p_value es menor a 0.05 por lo que es significativo, por lo que se acepta la hipótesis alterna $\mathrm{H} 2=\mathrm{La}$ estimulación intelectual incide en el trabajo colaborativo y las relaciones interpersonales de la UGEL 11.

Tabla 5. Coeficientes de la estimulación intelectual en las relaciones interpersonales y el trabajo colaborativo.

\begin{tabular}{|c|c|c|c|c|c|c|c|c|}
\hline \multicolumn{9}{|c|}{ Estimaciones de parámetro } \\
\hline & & \multirow{2}{*}{ Estimación } & \multirow{2}{*}{$\begin{array}{l}\text { Error } \\
\text { estándar }\end{array}$} & \multirow{2}{*}{ Wald } & \multirow{2}{*}{ gl } & \multirow{2}{*}{ Sig. } & \multicolumn{2}{|c|}{$\begin{array}{c}\text { Intervalo de } \\
\text { confianza al } 95 \%\end{array}$} \\
\hline & & & & & & & $\begin{array}{l}\text { Límite } \\
\text { inferior }\end{array}$ & $\begin{array}{l}\text { Límite } \\
\text { superior }\end{array}$ \\
\hline \multirow[t]{2}{*}{ Umbral } & [est_intelec $=1]$ & $-21,765$ & ,952 & 578,186 & 1 &, 000 & $-2,631$ & 1,100 \\
\hline & [est_intelec $=2]$ & $-19,392$ & ,949 & 532,171 & 1 &, 000 & $-2,252$ & 1,467 \\
\hline \multirow[t]{6}{*}{ Ubicación } & {$[$ Relac_intepr $=1]$} & $-20,643$ & 1,412 & 893,508 & 1 &, 000 & $-5,410$ & , 125 \\
\hline & [Relac_intepr=2] & $-22,343$ & 1,362 & & & . & $-4,903$ & ,436 \\
\hline & [Relac_intepr=3] & $0 \mathrm{a}$ & . & . & 0 & . & . & . \\
\hline & {$[$ trab_colab $=1]$} & ,220 & 1,184 & 1,203 & 1 &, 513 & $-1,021$ & 3,618 \\
\hline & {$[$ trab_colab $=2]$} & ,314 & 1,176 & 1,445 & 1 & ,463 &,- 892 & 3,720 \\
\hline & {$[$ trab_colab $=3]$} & $0 \mathrm{a}$ & . & . & 0 & . & . & . \\
\hline
\end{tabular}

Función de enlace: Logit.

a. Este parámetro está establecido en cero porque es redundante. 


\section{Prueba de hipótesis específica 3}

En el resultado del $\mathrm{R}$ del Pseudo cuadrado, se aprecia porcentualmente la influencia de la motivación en las relaciones interpersonales y trabajos colaborativos en los directivos de la UGEL
11-Cajatambo. El coeficiente de Nagelkerke hace referencia que la inconsecuencia de las relaciones interpersonales y el trabajo colaborativo es debido al 57,6\% del actuar de la motivación en las entidades educativas (ver Tabla 6).

Tabla 6. Resultado de regresión por Pseudo R-cuadrado, dimensión motivación.

\begin{tabular}{llllll}
\hline Cox y Snell &, 482 & Nagelkerke & ,576 & McFadden &, 395 \\
\hline
\end{tabular}

Función de enlace: Logit.

Respecto a la conducta de las relaciones interpersonales es complicación y presentando como coeficiente 1.017 y el trabajo colaborativo es inseguridad con $-23,645$ por lo que se puede afirmar que una mala práctica de motivación ocasionará bajas relaciones personales y trabajo colaborativo en los directivos de la UGEL 11 - Cajatambo, confirmándose esto con la prueba de Wald 876.537 y 503.573, respectivamente. (Ver Tabla 7).

Además, el p_value es menor a 0.05 por lo que se acepta la hipótesis alterna H3= La motivación/ inspiración tiene incidencia en las relaciones interpersonales y el trabajo colaborativo de los directivos de la UGEL 11 Cajatambo.

Tabla 7. Coeficientes de motivación en las relaciones interpersonales y el trabajo colaborativo.

\begin{tabular}{|c|c|c|c|c|c|c|c|c|}
\hline \multicolumn{9}{|c|}{ Estimaciones de parámetro } \\
\hline & & \multirow{2}{*}{ Estimación } & \multirow{2}{*}{$\begin{array}{c}\text { Error } \\
\text { estándar }\end{array}$} & \multirow{2}{*}{ Wald } & \multirow{2}{*}{ gl } & \multirow{2}{*}{ Sig. } & \multicolumn{2}{|c|}{$\begin{array}{c}\text { Intervalo de } \\
\text { confianza al 95\% }\end{array}$} \\
\hline & & & & & & & $\begin{array}{l}\text { Límite } \\
\text { inferior }\end{array}$ & $\begin{array}{l}\text { Límite } \\
\text { superior }\end{array}$ \\
\hline \multirow[t]{2}{*}{ Umbral } & {$[$ motiv_insp $=1]$} & $-22,732$ & ,964 & 503,573 & 1 &, 000 & $-1,156$ & 2,621 \\
\hline & {$[$ motiv_insp $=2]$} & $-23,287$ & 1,075 & 589,341 & 1 &, 000 & 1,179 & 5,395 \\
\hline \multirow[t]{6}{*}{ Ubicación } & [Relac_intepr=1] & $-20,287$ & 1,266 & 689,051 & 1 &, 000 & $-2,768$ & 2,195 \\
\hline & [Relac_intepr=2] & $-23,645$ & 1,273 & 876,537 & 1 &, 000 & $-3,424$ & 1,565 \\
\hline & [Relac_intepr=3] & 0a & . & . & 0 & . & . & . \\
\hline & {$[$ trab_colab $=1]$} & 1,017 & 1,105 & ,852 & 1 &, 553 & $-1,146$ & 3,185 \\
\hline & {$[$ trab_colab=2] } & ,489 & 1,146 & ,182 & 1 & ,443 & $-1,757$ & 2,734 \\
\hline & {$[$ trab_colab $=3]$} & $0 \mathrm{a}$ & . & . & 0 & . & . & . \\
\hline
\end{tabular}

Función d

a. Este parámetro está establecido en cero porque es redundante.e enlace: Logit. 


\section{Prueba de hipótesis específica 4}

$\mathrm{H} 4=\mathrm{La}$ influencia idealizada tiene incidencia en las relaciones interpersonales y el trabajo colaborativo de los directivos de UGEL 11.

En el resultado del R del Pseudo cuadrado, se observa la subordinación en porcentaje de la influencia idealizada en las relaciones interpersonal y trabajo colaborativo en los directivos de la UGEL 11 - Cajatambo. El coeficiente de Nagelkerke alude que la inconsecuencia de las relaciones interpersonales y el trabajo colaborativo se debe al $76,7 \%$ por el actuar de la influencia idealizada en las entidades educativas (ver Tabla 8).

Tabla 8. Resultado de regresión por Pseudo R-cuadrado, dimensión relaciones interpersonales

\begin{tabular}{llllll}
\hline \multicolumn{7}{c}{ Pseudo R cuadrado } \\
\hline Cox y Snell & ,663 & Nagelkerke &, 767 & McFadden &, 531 \\
\hline
\end{tabular}

Función de enlace: Logit.

En relación a las derivaciones se visualizan los coeficientes de la regresión en relación al trabajo colaborativo de nivel 1(bajo) y las relaciones interpersonales de nivel 1(bajo). Con respecto a la conducta del trabajo colaborativo es inseguridad con $-21,395$ y la relación interpersonal de malo con -22.521 por lo que a las malas prácticas de una influencia idealizada se tendrá bajas relaciones personales y trabajo colaborativo, dicha prueba se confirma con la prueba de Wald 502,835 y 489,083 (ver Tabla 9).

Tabla 9. Coeficientes de trabajo colaborativo en las relaciones interpersonales y el trabajo colaborativo.

\begin{tabular}{|c|c|c|c|c|c|c|c|c|}
\hline \multicolumn{9}{|c|}{ Estimaciones de parámetro } \\
\hline & & \multirow{2}{*}{ Estimación } & \multirow{2}{*}{$\begin{array}{l}\text { Error } \\
\text { estándar }\end{array}$} & \multirow{2}{*}{ Wald } & \multirow{2}{*}{ gl } & \multirow{2}{*}{ Sig. } & \multicolumn{2}{|c|}{$\begin{array}{c}\text { Intervalo de } \\
\text { confianza al 95\% }\end{array}$} \\
\hline & & & & & & & $\begin{array}{l}\text { Límite } \\
\text { inferior }\end{array}$ & $\begin{array}{l}\text { Límite } \\
\text { superior }\end{array}$ \\
\hline \multirow[t]{2}{*}{ Umbral } & [Influec_idealiz = 1] & $-21,521$ & ,904 & 502,835 & 1 & 000 & $-3,294$ & ,249 \\
\hline & [Influec_idealiz = 2] & 20,843 &, 889 & 394,898 & 1 &, 000 &,- 900 & 2,586 \\
\hline \multirow[t]{6}{*}{ Ubicación } & [Relac_intepr=1] & $-22,135$ & 1,177 & 809,293 & 1 &, 000 & $-4,442$ &, 171 \\
\hline & [Relac_intepr=2] & 24,169 & 1,122 & 489,083 & 1 &, 000 & $-3,369$ & 1,031 \\
\hline & [Relac_intepr=3] & $0 \mathrm{a}$ & . & . & 0 & . & . & . \\
\hline & {$[$ trab_colab $=1]$} & $-21,395$ & ,932 & 2,239 & 1 & 003 &,- 432 & 3,220 \\
\hline & {$[$ trab_colab=2] } & 337 & ,921 & ,478 & 1 & ,003 & $-1,169$ & 2,442 \\
\hline & {$[$ trab_colab=3] } & $0 \mathrm{a}$ & . & . & 0 & . & . & . \\
\hline
\end{tabular}

Función de enlace: Logit.

a. Este parámetro está establecido en cero porque es redundante. 


\section{Discusión}

Según los resultados obtenidos, se coincide con las conclusiones del autor Parreira et al. (2018) quien, en su estudio realizado en Brasil, valida una prueba de liderazgo la cual toma en consideración diferentes habilidades y es propuesta la evaluación de las competencias docentes. Obteniendo como resultado que una de las competencias más importantes para los docentes es el liderazgo transformacional, ya que los resultados obtenidos sugieren que es la más efectiva para alcanzar los objetivos de aprendizaje. Esta afirmación está relacionada con los resultados obtenidos en el estudio, ya que se comprobó que el liderazgo transformacional influye tanto en el trabajo colaborativo y las relaciones interpersonales, lo que implica una mejora en el logro de los objetivos planteados y un buen desarrollo de los proyectos educativos.

En este sentido, Rivera (2020) concluye que las prácticas de liderazgo que fueron implementadas como parte del programa estudiado, promueven el trabajo colaborativo, la reflexión sobre el desempeño y la práctica profesional. Afirmando lo planteado por la hipótesis general de estudio respecto al trabajo colaborativo, por otro lado, destaca la retroalimentación que implica el liderazgo transformacional. Asimismo, Aparicio y Sepúlveda (2019) bajo el concepto de qué la educación chilena ha tenido diversos procesos de cambios, exploran el discurso de los docente respecto a la experiencia que estos tienen en el trabajo colaborativo con sus colegas teniendo en cuenta los factores que facilitan y obstaculizan el mismo. Obteniendo como uno de los resultados que los docentes identifican la necesidad de apoyarse entre sus compañeros a fin de buscar estrategias comunes en contextos que les resultan difíciles o diferentes a lo usual, considerando beneficioso el trabajo colaborativo. Es por esto que es importante que exista cohesión social y cultura organizacional que considere estos aspectos dentro de la institución educativa, ya que según Rodríguez y Ossa (2014), quienes en su estudio realizado en Chile determinaron la percepción que tienen los profesores de educación especial y educación regular respecto al trabajo colaborativo en el contexto del programa de integración escolar. Encontrando que existen algunos grados de colaboración, sin embargo, también se aprecia la influencia de conceptos tradicionales y discriminadores entre las modalidades educación especial y regular.

Por su parte Echerri et al. (2019) exponen como objetivo comprobar el efecto que tiene el liderazgo transformacional como moderador del efecto negativo que produce el estrés laboral dentro de una institución educativa. Concluyendo que el liderazgo es un mitigador del estrés laboral por lo que los directores que tienen la capacidad de liderar de manera adecuada, garantizan que sus instituciones educativas cumplan los objetivos propuestos y sean menos afectas por el estrés laboral dentro de una institución educativa en México. El autor en su estudio resalta las dimensiones motivación/ inspiración, ya que, si el director de la institución educativa se implica en los objetivos comunes y mejora la capacidad de motivar al personal, encaminaría a su equipo hacia el logro de los objetivos propuestos.

De esta forma mejoraría el clima institucional $\mathrm{y}$ en consecuencia las relaciones interpersonales del personal de educación ya que, según el estudio realizado por Vásquez (2019), el cual tuvo como objetivo la determinación de la relación del clima institucional en las relaciones interpersonales de los profesores y el director en un colegio de Bagua grande, concluyó que existe una influencia entre el clima institucional y las relaciones interpersonales en los docentes y directivo. Según lo descrito, es 
importante que los directores educativos tengan desarrolladas sus habilidades, competencias y estrategias directivas con la finalidad de optimizar el clima organizacional dentro de la escuela y a la vez mejorar las relaciones interpersonales del personal educativo.

Así, siguiendo a Mendoza et al. (2019) recomienda realizar medidas correctivas en una escuela de Venezuela, a partir del objetivo planteado de analizar las habilidades comunicacionales como un elemento que permite optimizar las relaciones interpersonales entre los docentes y los directores en una institución educativa de Venezuela. Concluyeron quelos directivos educativos no aplican de forma correcta sus estrategias gerenciales, por lo que esto debilita las relaciones interpersonales del personal educativo de esta institución.

Por otro lado, los autores Leiva y Vásquez (2019) en su estudio realizado en Chile, resaltan la necesidad de que los directivos de dicha nación, realicen un proceso de acompañamiento a los docentes, teniendo en cuenta el liderazgo, destacando que el programa evaluado es fluctuante y existe una discrepancia entre los directivos y los docentes, ya que muchas veces en vez de tener un acompañamiento facilitador y colaborativo, los docentes mencionan que este es más de carácter intervencionista. Es así que se resalta que el liderazgo transformacional implica varios factores, entre estos se tiene que contemplar la motivación, inspiración, la estimulación intelectual que el líder o director plantea a los docentes a cargo, teniendo en cuenta a cada individuo, de no cumplirse con esto es muy probable que los programas planteados por la dirección no funcionen de manera óptima.

\section{CONCLUSIONES}

El liderazgo transformacional incide significativamente en las relaciones interpersonales y trabajo colaborativo en las instituciones educativas de la UGEL N ${ }^{\circ} 11$, Cajatambo. Si el director desarrolla adecuadamente su liderazgo, impactará de forma positiva en el trabajo colaborativo y las relaciones interpersonales del personal de educación.

Adicionalmente, la consideración individual incide significativamente en las relaciones interpersonales y trabajo colaborativo en las instituciones educativas de la UGEL $N^{\circ} 11$, Cajatambo. Es decir, si el director fomenta el respeto a la individualidad de cada integrante del equipo, impactará de forma positiva en el trabajo colaborativo y las relaciones interpersonales del personal de educación.

Por otra parte, la estimulación intelectual incide significativamente en las relaciones interpersonales y trabajo colaborativo en las instituciones educativas de la UGEL Nº11, Cajatambo. En consecuencia, si el director empodera y estimula a los integrantes de su equipo para que desarrollen sus habilidades intelectuales, impactará de forma positiva en el trabajo colaborativo y las relaciones interpersonales del personal de educación.

Es importante resaltar en estas líneas finales que la motivación incide significativamente en las relaciones interpersonales y trabajo colaborativo en las instituciones educativas de la UGEL $\mathrm{N}^{\circ} 11$, Cajatambo. Es decir, si el director tiene la capacidad de brindar motivación a su equipo, impactará de forma positiva en el trabajo colaborativo y las relaciones interpersonales del personal de educación.

Así, en definitiva, la influencia idealizada incide significativamente en las relaciones interpersonales y trabajo colaborativo en las instituciones educativas de la UGEL N${ }^{\circ} 11$, Cajatambo. Si el director brinda un propósito a su equipo, impactará de forma positiva en el trabajo colaborativo y las relaciones interpersonales del personal de educación. 


\section{REFERENCIAS}

Alqatawenah, A. S. (2018). Transformational leadership style and its relationship with change management. Bussines: Theory and Practice, 25. doi: https://doi.org/10.3846/btp.2018.03

Aparicio, C., y Sepúlveda, F. (2019). Trabajo colaborativo docente : nuevas perspectivas para el desarrollo docente

Avolio, B., y Bass, B. (2004). Multifactor Leadership Questionnaire.Third Edition Manual and Sampler Set. Mind Garden, Inc. doi: http:// dx.doi.org/10.1207/s1532754xjprr1602_2

Contreras, T. (2016). Liderazgo pedagógico, liderazgo docente y su papel en la mejora de la escuela: una aproximación teórica. Propósitos y Representaciones, 4(2), 231-284. doi:10.20511/ pyr2016.v4n 2.122

Dalton, M., Hoyle, D. y Watts, M. (2007). Relaciones humanas. (3a ed.). México: Thomson

Driscoll, M. y Vergara, A. (1997). Nuevas tecnologías y su impacto en la educación del futuro, en Pensamiento educativo, 21. Santiago de Chile, Pontificia Universidad Católica

Echerri, D., Santoyo, F., Rangel, M. Á., y Saldaña, C. (2019). Efecto modulador del liderazgo transformacional en el estrés laboral y la efectividad escolar percibida por trabajadores de educación básica de Ciudad Guzmán, Jalisco. Revista Iberoamericana de Investigación y Desarrollo, 9

Leiva, M., y Vásquez, C. (2019). Liderazgo pedagógico: de la supervisión al acompañamiento docente.Introducción. 225251

Madrigal, B. (2011). Liderazgo y aprendizaje. México: Mc Graw-Hill Interamericana Editores

Mendoza, J. L., Torrealba, M., y Montilla, A. (2019). Habilidades comunicativas como elemento optimizador de las relaciones interpersonales entre el personal directivo y los docentes de aula del liceo bolivariano "Héctor Rojas Meza" de Cabudare, Estado Lara, Venezuela. Pedagogía Universitaria, 17(2009), 111-122
Ministerio de Educación en Perú. (2018) Evaluación del Desempeño en Cargos Directivos de IE de Educación Básica - EDDir. Recuperado de: https://www.ugel01.gob.pe/wp-content/ uploads/2018/06/Charlas-Informativas-deEDDIR-04-07-18.pdf

Padilla, W. F., Pino, R. M., y Amaya, A. A. (2021). Factores que impactan en los criterios de éxito de los proyectos en Perú y Ecuador: el rol moderador de las competencias del director de proyecto. Información Tecnológica, 32(4), 133-146. https://doi.org/10.4067/s071807642021000400133

Parreira, A., Pestana, M. H., y Oliveira, P. (2018). Assessing educational leadership: A competence-complexity based test. Ensaio, 26(100), 890-910. https://doi.org/10.1590/ S0104-40362018002601559

Rivera, Y. (2020). Características de las prácticas de liderazgo pedagógico en programas de integración escolar que favorecen el trabajo colaborativo entre docentes. Perspectiva Educacional, 59(2), 27-44. https://doi. org/10.4151/07189729-vol.59-iss.2-art.1055

Rodríguez, F., y Ossa, J. (2014). Valoración del trabajo colaborativo entre profesores de escuelas básicas de Tomé, Chile. Estudios Pedagógicos, 40(2), 303-319

Rodríguez, M., Ordóñez, R., y López, A. (2020). La dirección escolar: Liderazgo pedagógico y mejora escolar. Revista de Investigación Educativa, 38(1), 275-292. doi:10.6018/ rie. 364581

Rosas, E. F. (2020). Liderazgo pedagógico directivo y desempeño docente en una Institución Educativa de Lima Metropolitana. REVISTA CONCIENCIA EPG, 1(1), 53-63. https://doi. org/10.32654/concienciaepg.1-1.5

Vásquez, M. (2019). Influencia del clima institucional en las relaciones interpersonales director y profesores en la Institución Educativa №16211 Bagua Grande. 0-2 